\title{
Microstructural changes of the whole brain in patients with comitant strabismus: evidence from a diffusion tensor imaging study
}

This article was published in the following Dove Press journal:

Neuropsychiatric Disease and Treatment

12 August 2016

Number of times this article has been viewed

\author{
Xin Huang ${ }^{1,2, *}$ \\ Hai-Jun $\mathrm{Li}^{3, *}$ \\ Ying Zhang' \\ De-Chang Peng ${ }^{3}$ \\ Pei-Hong $\mathrm{Hu}^{\prime}$ \\ Yu-Lin Zhong' \\ Fu-Qing Zhou ${ }^{3}$ \\ Yi Shao'
}

'Department of Ophthalmology, The First Affiliated Hospital of Nanchang University, Nanchang, ${ }^{2}$ Department of Ophthalmology, The First People's Hospital of Jiujiang City, Jiujiang,

${ }^{3}$ Department of Radiology, The First Affiliated Hospital of Nanchang University, Nanchang, Jiangxi, People's Republic of China

*These authors contributed equally to this work

Correspondence: Yi Shao

Department of Ophthalmology, The First Affiliated Hospital of Nanchang University, No 17, YongWaiZheng Street, DongHu District, Nanchang 330006,

Jiangxi, People's Republic of China

Tel/fax +86 79I 88692520

Email freebee99@।63.com

Fu-Qing Zhou

Department of Radiology, The First

Affiliated Hospital of Nanchang

University, No 17, YongWaiZheng Street,

DongHu District, Nanchang 330006,

Jiangxi, People's Republic of China

Tel +8679188695132

Emailfq.chou@yahoo.com
Objective: The aim of this study was to investigate the fractional anisotropy (FA) and mean diffusivity (MD) using a diffusion tensor imaging technique and whole-brain voxel-based analysis in patients with comitant strabismus.

Patients and methods: A total of 19 (nine males and ten females) patients with comitant strabismus and 19 age-, sex-, and education-matched healthy controls (HCs) underwent magnetic resonance imaging examination. Imaging data were analyzed using two-sample $t$-tests to identify group differences in FA and MD values. Patients with comitant strabismus were distinguishable from $\mathrm{HCs}$ by receiver operating characteristic curves.

Results: Compared with HCs, patients with comitant strabismus exhibited significantly decreased FA values in the brain regions of the left superior temporal gyrus and increased values in the bilateral medial frontal gyrus, right globus pallidus/brainstem, and bilateral precuneus. Meanwhile, MD value was significantly reduced in the brain regions of the bilateral cerebellum posterior lobe and left middle frontal gyrus but increased in the brain regions of the right middle frontal gyrus and left anterior cingulate.

Conclusion: These results suggest significant brain abnormalities in comitant strabismus, which may underlie the pathologic mechanisms of fusion defects and ocular motility disorders in patients with comitant strabismus.

Keywords: comitant strabismus, diffusion tensor imaging, mean diffusivity, fractional anisotropy, resting state

\section{Introduction}

Strabismus is a very common eye disease characterized by abnormal eye movements. According to $\mathrm{Fu}$ et al, ${ }^{1}$ the prevalence of strabismus was 108 out of 2,260 (5.0\%) eligible students in the Central China. Strabismus can be roughly divided into comitant strabismus and incomitant strabismus. Strabismus is not only a cosmetic disease but also causes severe damages to visual acuity (VA), binocular vision, and stereopsis. ${ }^{2}$ Surgery is currently the main treatment for strabismus. ${ }^{3}$

Strabismus demonstrates abnormal eye movements, which is accompanied by dysfunction of the cerebral cortex. A previous study showed that eye monosynaptic interhemisphere connections existed in strabismus cats. ${ }^{4}$ Other studies reported the abnormalities of visual cortex structure in infantile esotropic macaque monkeys. ${ }^{5,6}$ Another study demonstrated visual cortex suppression in patients with strabismus. ${ }^{7}$

Diffusion tensor imaging (DTI) is a widely used magnetic resonance imaging (MRI) modality that depicts water diffusion directionality as mean diffusivity (MD) and fractional anisotropy (FA). ${ }^{8}$ The MD value measures the total amount of diffusion within a 
voxel and provides the overall magnitude of water diffusion, while the FA value is a scalar value between 0 and 1 calculated from the eigenvalues $(\lambda 1, \lambda 2$, and $\lambda 3)$ of the diffusion tensor. It measures the overall directionality of water diffusion and reflects the complexity of cytoskeleton architecture, which restricts the intra- and extracellular water movement. ${ }^{9}$ The direction of water diffusion can indicate myelin sheath damage and tissue changes. Consequently, DTI has been applied to study various diseases, such as schizophrenia ${ }^{10}$ and stroke. ${ }^{11}$ DTI has also been used to evaluate brain microstructural changes of patients with strabismus amblyopia. Duan et $\mathrm{al}^{12}$ found that MD values were increased not only in optic radiation but also in certain brain regions of strabismus amblyopia patients with the DTI method. However, very few studies used the DTI strategy to investigate the microstructural changes in comitant strabismus. Here, to our knowledge, our study is the first to explore whole-brain microstructural changes in patients with comitant strabismus.

\section{Patients and methods Patients}

A total of 19 patients with comitant strabismus (nine males and ten females; four esotropia; and 15 exotropia) were recruited from the First Affiliated Hospital of University of South China and the Department of Ophthalmology, The First Affiliated Hospital of Nanchang University. The diagnostic criteria for comitant strabismus were as follows: 1) strabismus starting from birth; 2) stereovision defects (no visual fusion); 3) equal binocular corrected VA; and 4) with alternated cover, the experimental and strabismus angle were equal. Patients were excluded if they met any one of the following conditions: 1) acquired strabismus, incomitant strabismus, and concealed oblique; 2) eye diseases (infection, inflammation, and ischemic diseases); 3) history of eye surgeries (extraocular or intraocular surgeries); 4) psychiatric disorders (obsessive-compulsive disorder, anxiety disorder, schizophrenia, depression, etc), diabetes, cardiovascular diseases, and cerebral infarction diseases; and 5) addictions (eg, drugs and alcohol).

Nineteen healthy controls (HCs; nine males and ten females) with similar age, sex, and education status were also recruited from healthy volunteers from citizens of Nanchang, Jiangxi, People's Republic of China. All HCs met the following requirements: 1) no abnormalities in the brain parenchyma on cranial MRI; 2) no ocular diseases with uncorrected or corrected VA $>1.0 ; 3$ ) no psychiatric diseases (obsessive-compulsive disorder, anxiety disorder, schizophrenia, depression, etc); and 4) able to undergo MRI (eg, no cardiac pacemaker or implanted metal devices). All research methods followed the Declaration of Helsinki and were approved by the First Affiliated Hospital of Nanchang University Ethics Committee. All subjects participated voluntarily and were informed of the purposes, methods, and potential risks before signing an informed consent form.

\section{Data acquisition}

MRI scanning was performed using a 3T MR scanner (Trio, Siemens, Munich, Germany). Each subject underwent spin echo single-shot echo planar imaging with the following parameters: repetition time/echo time $=7,200 / 104 \mathrm{~ms}$, number of excitations $=2$, matrix $=128 \times 128$, field of view $=230 \times 230 \mathrm{~mm}$, slice number $=49$, slice thickness $=2.5 \mathrm{~mm}$, axial orientation, 64 nonlinear diffusion-weighting gradient directions with $b=1,000 \mathrm{~s} / \mathrm{mm}^{2}$, and additional image without diffusion weighting $\left(b=0 \mathrm{~s} / \mathrm{mm}^{2}\right)$.

\section{Data processing}

Diffuse tensor images were analyzed with voxel-based analysis of DTI ${ }^{13}$ and processed with Statistic Parametric Mapping 2 (SPM2; Wellcome Department of Cognitive Neurology, London, UK) and FMRIB Software Library (FSL) (Version 3.3; www.fmrib.ox.ac.uk/fsl) software. The analysis was performed according to a previous study. ${ }^{14}$

\section{Statistical analysis}

Analyses were performed using the SPSS 13.0 statistical software (SPSS Inc., Chicago, IL, USA) for Windows. Two-sample $t$-tests were used to compare differences of the FA and MD values between strabismus and $\mathrm{HC}$ groups in a voxel-based manner using the analysis of covariance, with age and sex as covariates to control the effect of age and sex. $P<0.001$ was considered statistically significant.

\section{Clinical data analysis}

The cumulative clinical measurements including the duration of the onset of disease and best-corrected VA were recorded.

\section{Results \\ Clinical data of subjects}

There were no obvious differences in weight $(P=0.958)$ and age $(P=0.986)$ between the patients with comitant strabismus and the HCs. The mean duration of strabismus was $27.42 \pm 9.04$ years. No significant differences were found in the best-corrected VA-right $(P=0.161)$ and the best-corrected VA-left $(P=0.750)$ (clinical data are shown in Table 1$)$. 
Table I Demographic information and clinical measurements for Stra and HCs

\begin{tabular}{|c|c|c|c|c|}
\hline & Stra & HCs & $t$ & $P$-value \\
\hline Male/female & $9 / 10$ & $9 / 10$ & N/A & $>0.99$ \\
\hline Age (years) & $27.42 \pm 9.04$ & $27.37 \pm 8.75$ & 0.018 & 0.986 \\
\hline Weight (kg) & $60.53 \pm 6.52$ & $60.42 \pm 5.71$ & 0.053 & 0.958 \\
\hline Handedness & I9R & I9R & N/A & $>0.99$ \\
\hline Exotropic and esotropic & $4 / 15$ & N/A & N/A & $\mathrm{N} / \mathrm{A}$ \\
\hline Duration of strabismus (years) & $27.42 \pm 9.04$ & $\mathrm{~N} / \mathrm{A}$ & N/A & $\mathrm{N} / \mathrm{A}$ \\
\hline Best-corrected VA-right & $1.07 \pm 0.10$ & $1.14 \pm 0.18$ & -1.430 & 0.161 \\
\hline Best-corrected VA-left & $1.09 \pm 0.14$ & $I .1 I \pm 0.16$ & -0.321 & 0.750 \\
\hline
\end{tabular}

Note: $P$-value $<0.05$, independent $t$-test $P$-value between Stra and HCs. Data are mean \pm SD.

Abbreviations: HCs, healthy controls; N/A, not applicable; Stra, comitant strabismus; VA, visual acuity.

\section{FA differences}

Compared with the HC group, the FA values were significantly decreased in the brain regions of the left superior temporal gyrus $(P<0.001)$ but increased in the areas of the bilateral medial frontal gyrus, right globus pallidus/ brainstem, and bilateral precuneus in the comitant strabismus group $(P<0.001$; Figure 1 and Table 2$)$. The mean values of altered FA values between the comitant strabismus and $\mathrm{HC}$ group are shown in Figure3A and Table 3.

\section{MD differences}

Compared to the $\mathrm{HC}$ group, the MD values were significantly decreased in the brain regions of the bilateral cerebellum posterior lobe and left middle frontal gyrus $(P<0.01)$ and increased in the brain regions of the right middle frontal gyrus and left anterior cingulate in the comitant strabismus group $(P<0.01$; Figure 2 and Table 4$)$.

The mean values of altered MD values between the comitant strabismus and $\mathrm{HC}$ groups are shown in Figure 3B and Table 5 .

\section{Receiver operating characteristic curve}

We proposed that the differences of the FA and MD values between the strabismus and $\mathrm{HC}$ groups might be useful diagnostic markers. To test this possibility, mean values of the FA and MD of different brain regions were extracted and used to analyze receiver operating characteristic (ROC) curves. The areas under the ROC curve for FA values were as follows: bilateral medial frontal gyrus (0.964), right globus pallidus/brainstem (0.939), and bilateral precuneus (0.958)

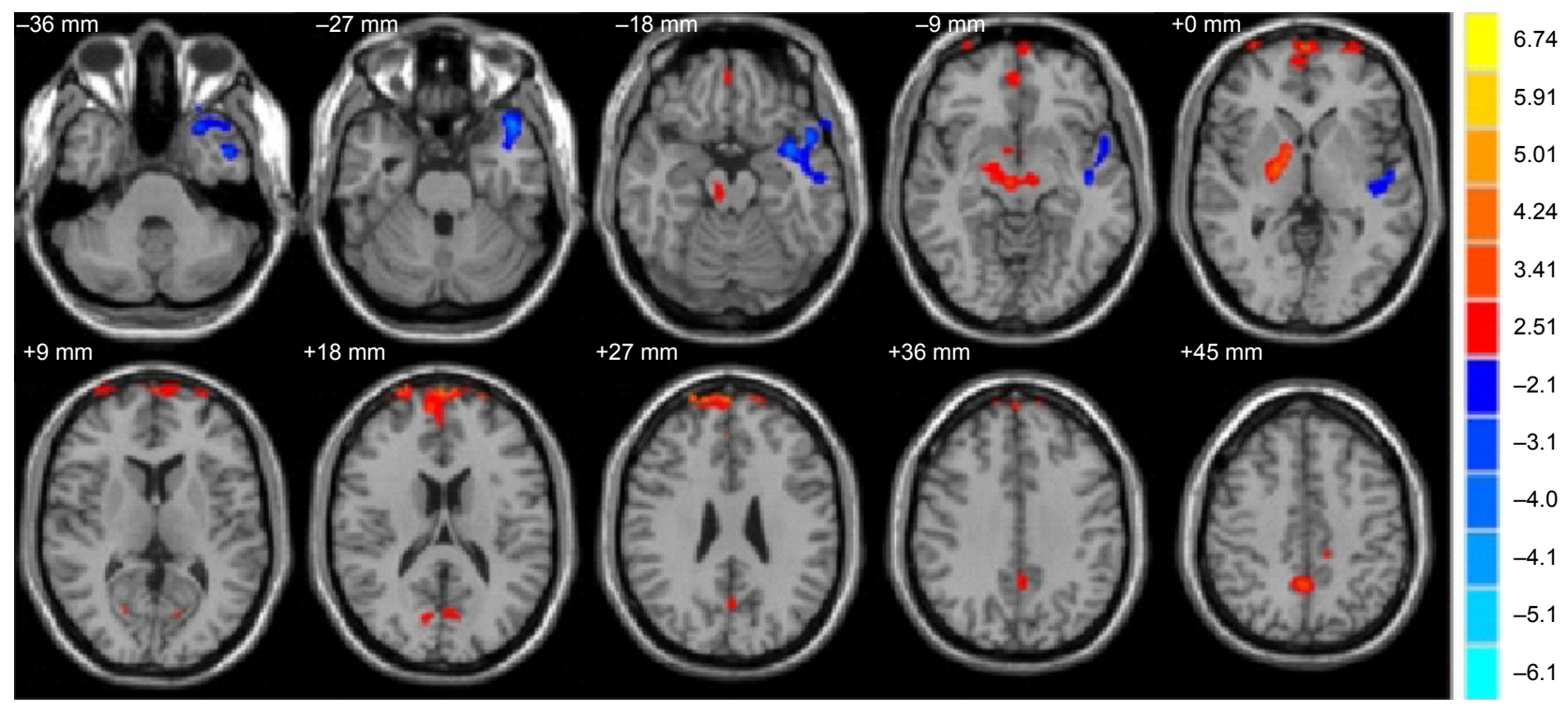

Figure I Significantly altered FA values in patients with Stra compared with HCs.

Notes: The significantly altered regions were located in the left superior temporal gyrus, bilateral medial frontal gyrus, right globus pallidus/brainstem, and bilateral precuneus. Red and blue areas denote regions with higher and lower FA, respectively. Statistical thresholds were set at $P<0.01$ (two sample $t$-test, corrected for false discovery rate) with a minimum cluster size of 75 voxels.

Abbreviations: FA, fractional anisotropy; HCs, healthy controls; Stra, comitant strabismus. 
Table 2 Brain regions with significant differences in the FA values between Stra group and HCs

\begin{tabular}{|c|c|c|c|c|c|c|}
\hline \multirow{2}{*}{$\begin{array}{l}\text { FA condition } \\
\text { Brain areas }\end{array}$} & \multicolumn{3}{|c|}{ Stra group and health controls } & \multicolumn{3}{|c|}{ MNI coordinates } \\
\hline & BA & $T$ values & Peak voxels & $x$ & $y$ & $\mathbf{z}$ \\
\hline \multicolumn{7}{|l|}{ Stra $<\mathrm{HCs}$} \\
\hline Left superior temporal gyrus & 21,22 , and 38 & -4.942 & 1,433 & -44 & 18 & -26 \\
\hline \multicolumn{7}{|l|}{ Stra $>\mathrm{HCs}$} \\
\hline Bilateral medial frontal gyrus & 10 and $I I$ & 5.790 & 1,494 & 2 & 66 & 24 \\
\hline Right globus pallidus/brainstem & 18 and 30 & 4.578 & 342 & 16.5 & -72 & 15 \\
\hline Bilateral precuneus & 7 and 31 & 3.919 & 348 & -10 & -42 & 56 \\
\hline
\end{tabular}

Note: Multiple comparisons using GRF theory (voxel-wise $P<0.01$ and cluster-wise $P<0.05$ corrected).

Abbreviations: BAs, Brodmann areas; FA, fractional anisotropy; GRF, Gaussian random field; HCs, healthy controls; MNI, Montreal Neurological Institute; Stra, comitant strabismus.

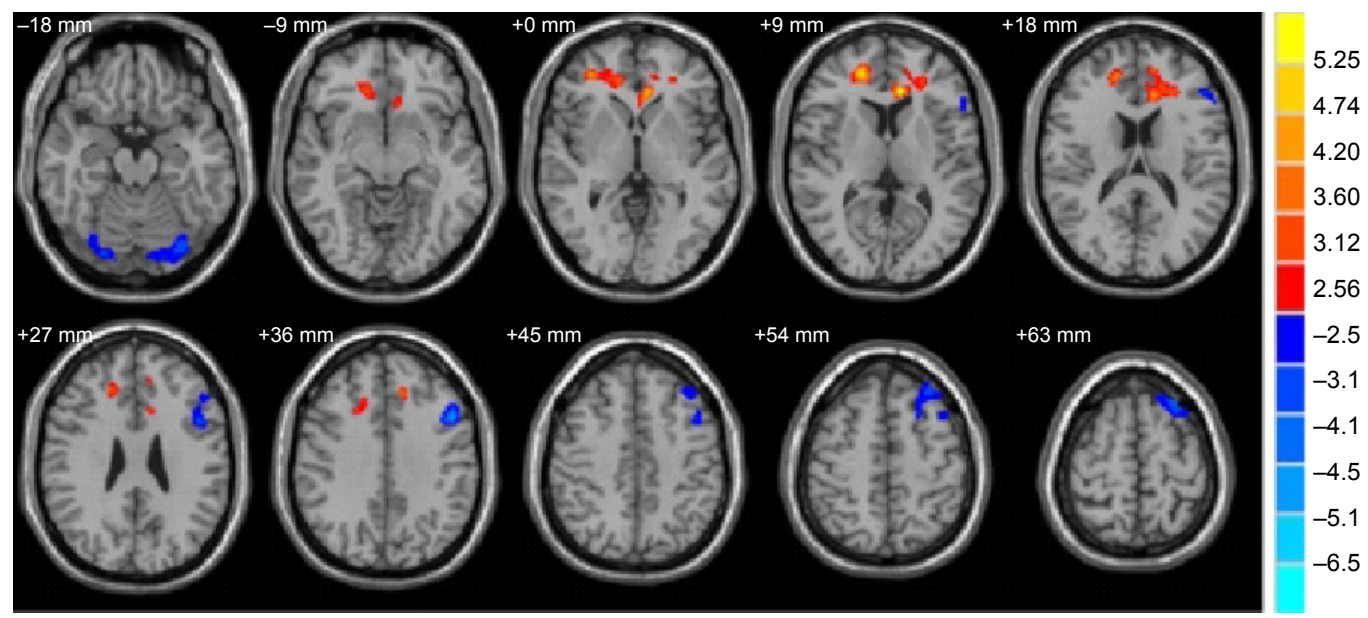

Figure 2 Significantly altered MD values in patients with Stra compared with HCs.

Notes: The significantly altered regions were located in the bilateral cerebellum posterior lobe, left middle frontal gyrus, right middle frontal gyrus, and left anterior cingulate. Red and blue areas denote regions with higher and lower MD, respectively. Statistical thresholds were set at $P<0.01$ (two sample $t$-test, corrected for false discovery rate) with a minimum cluster size of 75 voxels.

Abbreviations: HCs, healthy controls; MD, mean diffusivity; Stra, comitant strabismus.

A

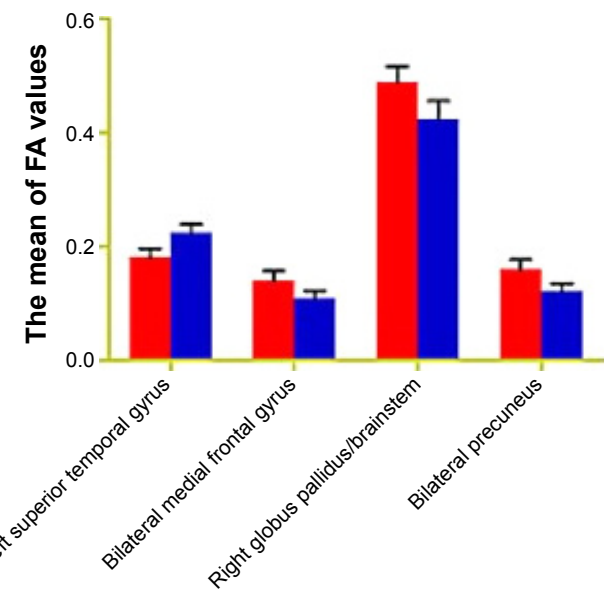

Altered FA regions
B

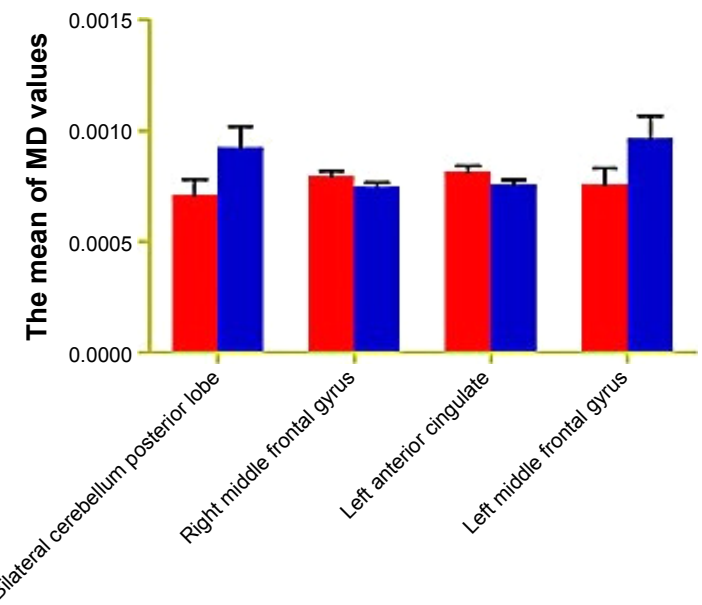

Altered MD regions

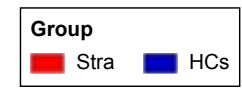

Figure 3 The mean values of altered MD and FA values between the Stra and HCs.

Notes: (A) Significantly altered FA values in patients with Stra. The significantly altered regions were located in the the left superior temporal gyrus, bilateral medial frontal gyrus, right globus pallidus/brainstem and bilateral precuneus. (B) Significantly altered MD values in patients with Stra. The significantly altered regions were located in the bilateral cerebellum posterior lobe, left middle frontal gyrus, right middle frontal gyrus and left anterior cingulate. Data are mean \pm SD.

Abbreviations: FA, fractional anisotropy; HCs, healthy controls; MD, mean diffusivity; Stra, comitant strabismus. 
Table 3 Significant differences in the FA values between Stra group and HCs

\begin{tabular}{lllll}
\hline FA & Stra & HCs & t & P-value \\
\hline Left superior temporal gyrus & $0.18 \pm 0.02$ & $0.22 \pm 0.02$ & -8.741 & $<0.001$ \\
Bilateral medial frontal gyrus & $0.14 \pm 0.02$ & $0.11 \pm 0.01$ & 6.535 & $<0.001$ \\
Right globus pallidus/brainstem & $0.49 \pm 0.03$ & $0.42 \pm 0.03$ & 6.712 & $<0.001$ \\
Bilateral precuneus & $0.16 \pm 0.02$ & $0.12 \pm 0.01$ & 7.553 & $<0.001$ \\
\hline
\end{tabular}

Note: $P$-value $<0.05$, independent $t$-test $P$-value between Stra and HCs. Data are mean $\pm S D$.

Abbreviations: FA, fractional anisotropy; HCs, healthy controls; Stra, comitant strabismus.

(Figure 4A). The areas under the ROC curve for MD values were as follows: right middle frontal gyrus (0.931) and left anterior cingulate (0.978) (Figure 4B).

\section{Discussion}

Our study is the first to evaluate whole-brain microstructural changes of FA and MD values in patients with comitant strabismus using a DTI approach. The FA is markedly sensitive to microstructural changes of white matter (WM), while the MD may help to better understand how the diffusion tensor is changing. The reduction of the FA values indicates WM neuropathology. Increased tissue water will lead to an increase in the MD values, and the decreased MD value may indicate cell proliferation. ${ }^{15}$ We found FA values remarkably decreased in the brain regions of the left superior temporal gyrus but increased in the areas of the bilateral medial frontal gyrus, right globus pallidus/brainstem, and bilateral precuneus. Meanwhile, the MD values were significantly decreased in the brain regions of the bilateral cerebellum posterior lobe and left middle frontal gyrus and increased in the brain regions of the right middle frontal gyrus and left anterior cingulate.

The superior temporal gyrus located in the temporal lobe of the human brain is involved in the processing of language. ${ }^{16}$ A previous study has shown that the superior temporal area controls the representation of three-dimensional structures and shapes. ${ }^{17}$ It has been well known that patients with strabismus often manifest dysfunction of fusion and stereopsis. ${ }^{18}$ Yan et $\mathrm{al}^{14}$ found that patients with comitant exotropia had smaller WM volumes in the right inferior temporal gyrus. In agreement with these findings, we found that the FA value was decreased in left superior temporal gyrus in comitant strabismus, which reflected abnormalities of WM fibers in these areas. These results suggest that comitant strabismus may cause dysfunction of the left superior temporal gyrus.

The medial frontal cortex is involved in the control, monitor, and selection of behaviors. The supplementary eye field located in the medial frontal cortex is involved in the execution of ocular movements. ${ }^{19}$ A previous study demonstrated that the frontal eye field (FEF) played an important role in controlling eye movements of monkeys. ${ }^{20}$ Another research reported that the FEF was responsible for rapid or saccadic ocular movements. ${ }^{21}$ Moreover, the FEF was shown to be in charge of sustained attention. ${ }^{22}$ Yan et a ${ }^{14}$ found that patients with comitant exotropia had smaller WM volumes in right frontal lobe/sub-gyral. Additionally, Chan et $\mathrm{al}^{23}$ observed increased gray matter volume in the FEF of adults strabismus. Consistent with these findings, we found that the MD values were significantly increased in the brain regions of the right middle frontal gyrus in patients with comitant strabismus, suggesting the dysfunction of WM fibers in these areas. Furthermore, we found that the mean area of the MD values of the right middle frontal gyrus was 0.931 in the ROC curve. Therefore, we speculated that comitant strabismus possibly caused abnormalities of WM fibers in right middle frontal

Table 4 Brain regions with significant differences in the MD values between Stra group and HCs

\begin{tabular}{|c|c|c|c|c|c|c|}
\hline \multirow{2}{*}{$\begin{array}{l}\text { MD condition } \\
\text { Brain areas }\end{array}$} & \multicolumn{3}{|c|}{ Stra group and health controls } & \multicolumn{3}{|c|}{ MNI coordinates } \\
\hline & BA & $T$ values & Peak voxels & $x$ & y & $\mathbf{z}$ \\
\hline \multicolumn{7}{|l|}{ Stra $<\mathrm{HCs}$} \\
\hline Bilateral cerebellum posterior lobe & - & -3.756 & $\mathrm{I}, 434$ & -26 & -52 & -52 \\
\hline Left middle frontal gyrus & 4,9 , and 46 & -5.100 & $\mathrm{I}, 200$ & -48 & 14 & 36 \\
\hline \multicolumn{7}{|l|}{ Stra $>\mathrm{HCs}$} \\
\hline Right middle frontal gyrus & 32 and 9 & 5.409 & 871 & 20 & 46 & 8 \\
\hline Left anterior cingulate & 32 and 24 & 5.872 & 913 & -10 & 32 & 10 \\
\hline
\end{tabular}

Note: Multiple comparisons using GRF theory (voxel-wise $P<0.01$ and cluster-wise $P<0.05$ corrected).

Abbreviations: BAs, Brodmann areas; GRF, Gaussian random field; HCs, healthy controls; MD, mean diffusivity; MNI, Montreal Neurological Institute; Stra, comitant strabismus. 
Table 5 Significant differences in the MD values between Stra group and HCs

\begin{tabular}{lllll}
\hline MD & Stra & HCs & $t$ & P-value \\
\hline Bilateral cerebellum posterior lobe & $0.0007 I \pm 0.00007 \mathrm{I}$ & $0.00093 \pm 0.000094$ & -7.997 & $<0.001$ \\
Right middle frontal gyrus & $0.0008 \pm 0.000025$ & $0.00075 \pm 0.00002$ & 6.558 & $<0.00$ I \\
Left anterior cingulate & $0.0008 I \pm 0.000028$ & $0.00076 \pm 0.000020$ & 7.18 I & $<0.00$ I \\
Left middle frontal gyrus & $0.00076 \pm 0.00007 \mathrm{I}$ & $0.00097 \pm 0.00010$ & -7.268 & $<0.00 \mathrm{I}$ \\
\hline
\end{tabular}

Note: $P$-value $<0.05$, independent $t$-test $P$-value between Stra and HCs. Data are mean $\pm S D$.

Abbreviations: $\mathrm{HCs}$, healthy controls; MD, mean diffusivity; Stra, comitant strabismus.

gyrus, which may explain the impairment of regular eye movements in the patients with comitant strabismus.

Interestingly, we found that the FA values were markedly increased in the area of the bilateral medial frontal gyrus, and the MD values were decreased in the left middle frontal gyrus in patients with comitant strabismus. An increase in the FA values in the bilateral medial frontal gyrus may reflect the WM alterations in these areas. The decreased MD values in the left middle frontal gyrus may indicate cell proliferation defects in these areas. Moreover, the mean area of the FA of the bilateral medial frontal gyrus was 0.964 . These changes may suggest a functional reorganization in the FEF in patients with comitant strabismus.

The cerebellum is involved in the control of movements ${ }^{24}$ and the execution of cognition ${ }^{25}$ and language. ${ }^{26}$ Moreover, a previous study demonstrated that the cerebellum was involved in the execution of eye and hand movements. ${ }^{27}$ Another study reported that activation of cerebellar vermis was related to visually guided saccades. ${ }^{28}$ Joshi and $\operatorname{Das}^{29}$ found that the posterior interposed nucleus in the cerebellum participated in conjugating eye movements in strabismic monkeys. In our study, we observed that the MD values were significantly decreased in the brain regions of the bilateral cerebellum posterior lobe in patients with comitant strabismus, which may reflect abnormalities of microstructural changes in these areas. We speculated that comitant strabismus possibly lead to pathological microstructural changes in the bilateral cerebellum posterior lobe, which manifested in the impairment of regular eye movements in the patients with comitant strabismus.

The cingulate cortex, as a part of the limbic cortex, has many functions, such as pain, ${ }^{30}$ depression, ${ }^{31}$ and anxiety disorder. ${ }^{32}$ Yan et $\mathrm{a}^{14}$ found that WM volumes were decreased in the right cingulate gyrus in patients with adult strabismus. In our study, we observed that the MD values were significantly increased in the brain regions of the left anterior cingulate in patients with comitant strabismus, which suggested abnormal microstructural changes in the left anterior cingulate. Furthermore, the mean area of the MD values
A

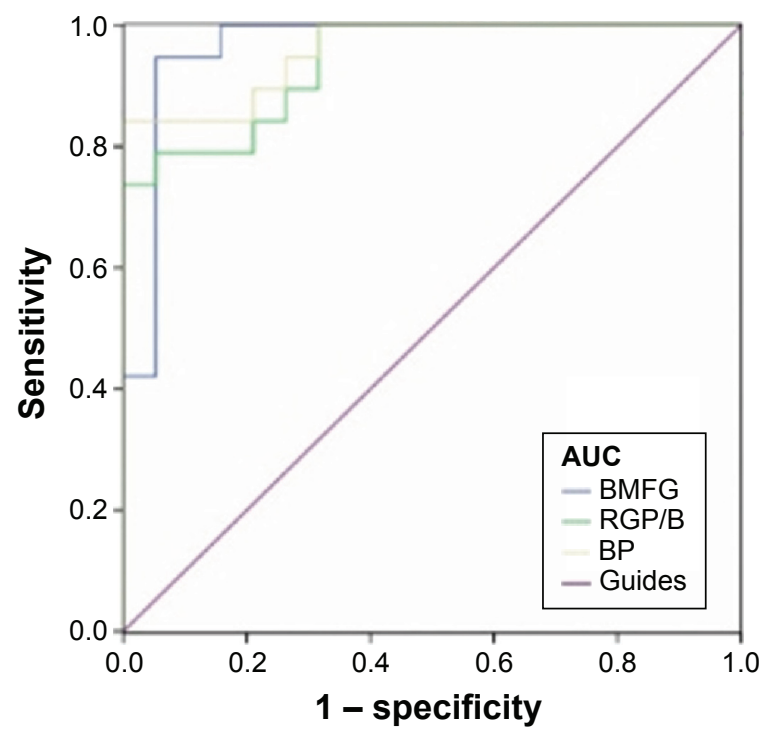

B

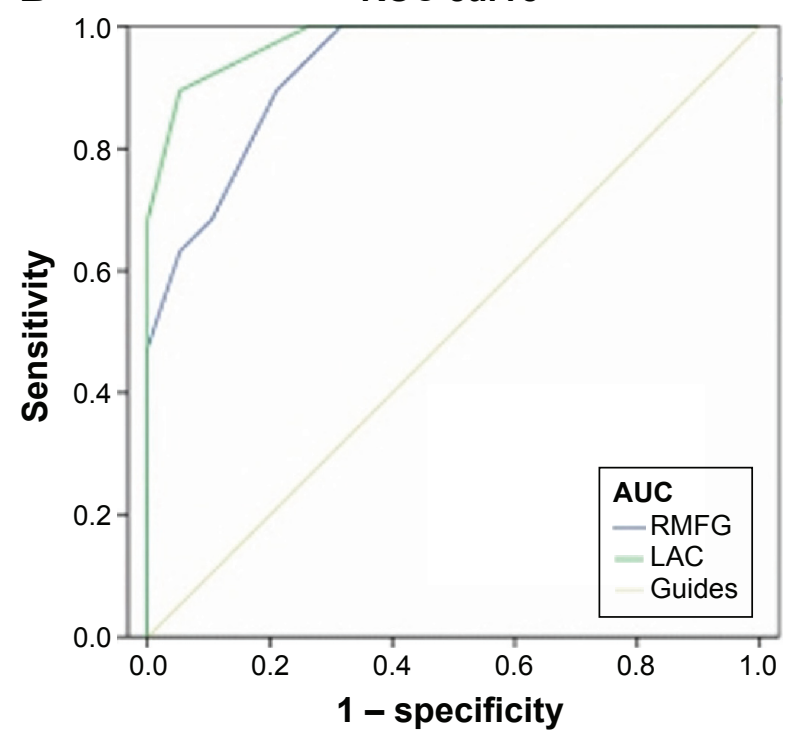

Figure 4 ROC curve analysis of the mean $F A$ and $M D$ values for altered brain regions.

Notes: (A) The AUCs for FA values: BMFG (0.964), RGP/B (0.939), and BP (0.958). (B) The AUCs for MD values: RMFG (0.93I) and LAC (0.978).

Abbreviations: AUCs, area under the ROC curves; BMFG, bilateral medial frontal gyrus; BP, bilateral precuneus; FA, fractional anisotropy; LAC, left anterior cingulated; RMFG, right middle frontal gyrus; MD, mean diffusivity; ROC, receiver operating characteristic; RGP/B, right globus pallidus/brainstem. 
of the left anterior cingulate was 0.978 in the ROC curve. Therefore, we surmised that the comitant strabismus might lead to the dysfunction of the left anterior cingulate.

\section{Conclusion}

Our study showed that patients with comitant strabismus had microstructural changes of WM in many brain regions and provided important information to understand the underlying neural mechanisms of the fusion defects and ocular motility disorders in patients with comitant strabismus. However, there are some limitations to our study. First of all, the sample size is small, and we did not consider different clinical outcomes of the strabismus, such as exotropia and esotropia. Future research should distinguish between different types of strabismus to more accurately assess brain activities and functional changes.

\section{Acknowledgments}

This study was supported by the National Natural Science Foundation of China (81400372, 81560285, and 81160118). This was not an industry-supported study.

\section{Disclosure}

The authors report no conflicts of interest in this work.

\section{References}

1. Fu J, Li SM, Liu LR, et al. Prevalence of amblyopia and strabismus in a population of 7th-grade junior high school students in Central China: the Anyang Childhood Eye Study (ACES). Ophthalmic Epidemiol. 2014; 21(3):197-203.

2. Feng L, Zhou J, Chen L, Hess RF. Sensory eye balance in surgically corrected intermittent exotropes with normal stereopsis. Sci Rep. 2015;5:13075.

3. Ogüt MS, Onal S, Demirtas S. Adjustable suture surgery for correction of various types of strabismus. Ophthalmic Surg Lasers Imaging. 2007;38(3):196-202.

4. Alekseenko SV, Shkorbatova PY, Toporova SN. Interhemisphere connections of the visual cortex in cats with bilateral strabismus. Neurosci Behav Physiol. 2006;36(9):1015-1019.

5. Tychsen L, Burkhalter A. Neuroanatomic abnormalities of primary visual cortex in macaque monkeys with infantile esotropia: preliminary results. J Pediatr Ophthalmol Strabismus. 1995;32(5):323-328.

6. Wong AM, Burkhalter A, Tychsen L. Suppression of metabolic activity caused by infantile strabismus and strabismic amblyopia in striate visualcortex of macaque monkeys. J AAPOS. 2005;9(1):37-47.

7. Chen VJ, Tarczy-Hornoch K. Functional magnetic resonance imaging of binocular interactions in visual cortex in strabismus. $J$ Pediatr Ophthalmol Strabismus. 2011;48(6):366-374.

8. Beaulieu C. The basis of anisotropic water diffusion in the nervous system - a technical review. NMR Biomed. 2002;15(7-8):435-455.

9. Pierpaoli C, Basser PJ. Toward a quantitative assessment of diffusion anisotropy. Magn Reson Med. 1996;36(6):893-906.

10. Sun Y, Chen Y, Lee R, Bezerianos A, Collinson SL, Sim K. Disruption of brain anatomical networks in schizophrenia: a longitudinal, diffusion tensor imaging based study. Schizophr Res. 2016;171(1-3):149-157.
11. Jang SH, Yi JH, Choi BY, et al. Changes of the corticospinal tract in the unaffected hemisphere in stroke patients: a diffusion tensor imaging study. Somatosens Mot Res. 2016;18:1-7.

12. Duan Y, Norcia A, Yeatman J, et al. A surveyof the integrity of major white matter tracts in strabismic amblyopia. $J$ Vis. 2015;15(12):650.

13. Shu N, Li J, Li K, Yu C, Jiang T. Abnormal diffusion of cerebral white matter in early blindness. Hum Brain Mapp. 2009;30(1):220-227.

14. Yan X, Lin X, Wang Q, et al. Dorsal visual pathway changes in patients with comitant extropia. PLoS One. 2010;5(6):e10931.

15. Alexander AL, Lee JE, Lazar M, et al. Diffusion tensor imaging of the brain. Neurotherapeutics. 2007;4(3):316-329.

16. Kovelman I, Wagley N, Hay JS, et al. Multimodal imaging of temporal processing in typical and atypical language development. Ann N Y Acad Sci. 2015;1337:7-15.

17. Mysore SG, Vogels R, Raiguel SE, Todd JT, Orban GA. The selectivity of neurons in the macaque fundus of the superior temporal area for three-dimensional structure from motion. $J$ Neurosci. 2010;30(46): 15491-15508.

18. Feng X, Zhang X, Jia Y. Improvement in fusion and stereopsis following surgery for intermittent exotropia. J Pediatr Ophthalmol Strabismus. 2015;52(1):52-57.

19. Stuphorn V. The role of supplementary eye field in goal-directed behavior. J Physiol Paris. 2015;109(1-3):118-128.

20. Thompson KG, Hanes DP, Bichot NP, Schall JD. Perceptual and motor processing stages identified in the activity of macaque frontal eye field neurons during visual search. J Neurophysiol. 1996;76(6): 4040-4055.

21. Hanes DP, Wurtz RH. Interaction of the frontal eye field and superior colliculus for saccade generation. J Neurophysiol. 2001;85(2):804-815.

22. Esterman M, Liu G, Okabe H, Reagan A, Thai M, DeGutis J. Frontal eye field involvement in sustaining visual attention: evidence from transcranial magnetic stimulation. Neuroimage. 2015;111:542-548.

23. Chan ST, Tang KW, Lam KC, Chan LK, Mendola JD, Kwong KK. Neuroanatomy of adult strabismus: a voxel-based morphometric analysis of magnetic resonance structural scans. Neuroimage. 2004; 22(2):986-994.

24. Paulin MG. The role of the cerebellum in motor control and perception. Brain Behav Evol. 1993;41(1):39-50.

25. Stoodley CJ. The cerebellum and cognition: evidence from functional imaging studies. Cerebellum. 2012;11(2):352-365.

26. Murdoch BE. The cerebellum and language: historical perspective and review. Cortex. 2010;46(7):858-868.

27. Nitschke MF, Arp T, Stavrou G, Erdmann C, Heide W. The cerebellum in the cerebro-cerebellar network for the control of eye and hand movements - an fMRI study. Prog Brain Res. 2005;148:151-164.

28. Hayakawa Y, Nakajima T, Takagi M, Fukuhara N, Abe H. Human cerebellar activation in relation to saccadic eye movements: a functional magnetic resonance imaging study. Ophthalmologica. 2002;216(6): 399-405.

29. Joshi AC, Das VE. Muscimol inactivation of caudal fastigial nucleus and posterior interposed nucleus in monkeys with strabismus. $J \mathrm{Neu}$ rophysiol. 2013;110(8):1882-1891.

30. Boccard SG, Pereira EA, Moir L, et al. Deep brain stimulation of the anterior cingulate cortex: targeting the affective component of chronicpain. Neuroreport. 2014;25(2):83-88.

31. Onoda K, Yamaguchi S. Dissociative contributions of the anterior cingulate cortex to apathy and depression: topological evidence from resting-state functional MRI. Neuropsychologia. 2015;77:10-18.

32. Shinoura N, Yamada R, Tabei Y, et al. The right dorsal anterior cingulate cortex may play a role in anxiety disorder and visual function. Neurol Res. 2013;35(1):65-70. 


\section{Publish your work in this journal}

Neuropsychiatric Disease and Treatment is an international, peerreviewed journal of clinical therapeutics and pharmacology focusing on concise rapid reporting of clinical or pre-clinical studies on a range of neuropsychiatric and neurological disorders. This journal is indexed on PubMed Central, the 'PsycINFO' database and CAS,

and is the official journal of The International Neuropsychiatric Association (INA). The manuscript management system is completely online and includes a very quick and fair peer-review system, which is all easy to use. Visit http://www.dovepress.com/testimonials.php to read real quotes from published authors.

Submit your manuscript here: http://www.dovepress.com/neuropsychiatric-disease-and-treatment-journal 